\title{
Comunicación publicitaria en medios digitales
}

La gestión de la responsabilidad social en las Organizaciones del Tercer Sector Advertising communication in digital media

The management of social responsibility in Third Sector Organizations

\section{Comunicação publicitária em mídia digital}

\section{A gestão da responsabilidade social em Organizações do Terceiro Setor}

\section{HERLINDA ORTIZ RODRÍGUEZ}

herlinda.ortiz@anahuac.mx - Universidad Anáhuac México, Campus Norte, México.

ORCID: https://orcid.org/0000-0001-7059-8931

Fecha de recepción: 1 de febrero de 2020

Fecha de aceptación: 23 de marzo de 2020

\section{RESUMEN}

La gestión de la Responsabilidad Social Organizacional (RSO) dentro de las Organizaciones del Tercer Sector (OTS) permite que se proyecten como organizaciones éticas y congruentes comprometidas con sus públicos, el entorno y la comunidad. La comunicación publicitaria en medios digitales favorece una adecuadagestión estratégica dela RSO para sensibilizar a sus públicos yobtener su apoyo. En esta investigación se analiza si las OTS de México, Chile, España e Inglaterra utilizan la comunicación publicitaria en sus páginas web para dar a conocer el modo en que asumen la RSO. Se miden tres variables de la RSO: comportamiento ético, medio ambiente y cambio climático y contribución a la comunidad, mediante un análisis de contenido. Los resultados muestran que las OTS de Inglaterra son las que más utilizan este tipo de comunicación publicitaria para dar a conocer sus acciones de RSO en comparación con las de México. No obstante, las organizaciones de los cuatro países tienen retos importantes para fortalecer su RSO y su comunicación 
publicitaria digital. Principalmente deben comprender quela correcta gestión de RSO y de la comunicación publicitaria en medios digitales fortalece su reputación y confianza con sus públicos.

PALABRAS CLAVE: públicos, comunicación publicitaria, Responsabilidad Social Organizacional, medios digitales, Organización del Tercer Sector.

\section{ABSTRACT}

Themanagement of the OrganizationalSocial Responsibility (OSR) in Third Sector Organizations (TSO) allows them to present themselves as ethical and coherent organizations engaged with their audiences, environments and community. Advertising communication in digital media allows proper strategic management of the RSO, in order to sensitize audiences and obtain their support. This inquiry analyses whether TSO from Mexico, Chile, Spain and England use advertising communication to make their OSR public in their websites. Three OSR variables aremeasured:ethicalbehaviour,environmentand climatechange and contribution to the community bycontent analysis. The results show that TSO from England are the ones that use advertising communication the most for making their OSR actions public, compared with thosefrom Mexico. Nevertheless, the organizations from thefour countries haveimportant challengesto face in order to strengthen their OSR and their digital advertising communication. Mainly, they must understand that the correct management of the OSR and advertising communication on digital media strengthen their reputation and trust with their audiences.
KEYWORDS: public, publicity communication, Organizational Social Responsibility, digital media, Third Sector Organization.

\section{RESUMO}

A gestão da Responsabilidade Social Organizacional (RSO) nas Organizações do Terceiro Setor (OTS) permite que elas se projetem como organizações éticas e congruentes, comprometidas com o público, o meio ambiente e a comunidade. A comunicação publicitária em mídia digital permite um adequado gerenciamento estratégico da RSO para sensibilizar seus públicos e obter apoio. Nesta pesquisaéanalisadoseOTS de México, Chile, Espanha e Inglaterra utilizam a comunicação publicitaria para divulgar como elas assumem a RSO através do seu site. Três variáveis da RSO são medidas: comportamento ético, meio ambiente e mudança climática e contribuição para a comunidade através da análise de conteúdo. Os resultados mostram que as OTS na Inglaterra são as que mais usam a comunicação publicitária para divulgar suas ações de RSO em comparação com as do México. No entanto, as organizações nos quatro países enfrentam desafios significativos no fortalecimento da sua RSO e sua comunicação publicitária digital. Principalmente, devem entender que o gerenciamento correto da RSO e a comunicação publicitária em meios digitais fortalecem sua reputação e confiança com seus públicos.

PALAVRAS-CHAVE: público, comunicação publicitária, Responsabilidade Social Organizacional, mídia digital, Organização do Terceiro Setor. 


\section{INTRODUCCIÓN}

En la actualidad, las Organizaciones del Tercer Sector (OTS) han tomado un papel protagónico como actores dinámicos y activos que se ocupan de diseñar acciones que buscan dar respuesta a distinto tipo de problemas sociales a los que se enfrentan los países y que sus ciudadanos tengan una mejor calidad de vida. No obstante, y si bien es cierto que las OTS buscan contribuir en la solución de problemas que afectan a los sectores más vulnerables de la sociedad, las propias organizaciones atraviesan diferentes retos. De acuerdo con Aguilar (2006), algunos de esos retos son: incrementar sus niveles de eficacia y credibilidad; diseñar, gestionar y evaluar políticas públicas; mejorar la profesionalización de la organización y de su personal; elevar la calidad e impacto de la acción; encontrar nuevas formas de financiamiento; garantizar la transparencia y rendición decuentas; superar los prejuicios ylas diferencias internas para hacer un frente común; elevar el nivel de presencia y visibilidad; cambiar la forma de relación con el gobierno o las instituciones del Estado; mejorar la relación con la sociedad; tender puentes con otros sectores y elevar el nivel de las relaciones constituidas.

Desde un punto de vista estratégico, es esencial que estas estructuras organizacionales resuelvan retos como los que fueron mencionados y que fortalezcan, en primer lugar, sus procesos de funcionamiento y comunicación interna. A partir de allí podrán definir las estrategias de comunicación publicitaria digital enfocadas a la Responsabilidad Social Organizacional (RSO) y alcanzar los objetivos propuestos. Las OTS se caracterizan por tener fines altruistas y un carácter relacional, ya que para alcanzar sus objetivos sociales requieren del apoyo incondicional de sus públicos. Dicho carácter relacional, en palabras de Donati (1997), significa hacer comunidad o hacer sociedad; es decir, establecer relaciones colaborativas y de confianza con sus públicos estratégicos, quienes hoy les exigen un mayor compromiso en sus acciones de RSO.

La RSO aplicada a las OTS debe ser entendida como un acto voluntario en el desarrollo de sus acciones estratégicas tendientes a beneficiar a sus públicos, a la sociedad y a los distintos entornos con los que interactúan. La creación de acciones enfocadas en la RSO implica construir estrategias reales y congruentes alineadas con los objetivos sociales de las OTS. En ese marco, el aprovechamiento de los medios digitales -como el uso de la comunicación publicitaria digital y la página web institucional-resulta esencial para que la comunicación genere lazos con los públicos de su interés, se establezcan vínculos de confian$\mathrm{za}$, se fortalezcan la reputación organizacional y, de esa manera, se obtengan apoyos y la comprensión necesaria para hacer frente a los distintos retos que se atraviesan.

En base a lo mencionado, el objetivo de la investigación estuvo centrado en comparar el nivel y el uso de la comunicación publicitaria digital de 40 OTS de México, Chile, España e Inglaterra para dar a conocer sus acciones de RSO. En 
tal sentido, se realizó un análisis de contenido de la comunicación publicada en la página web institucional de estas organizaciones y se buscó entender cuáles organizaciones aprovechan de forma efectiva su página web institucional para comunicarse con sus públicos. Esas acciones de comunicación publicitaria digital enfocadas a la RSO se midieron a través de tres principales variables: comportamientoético, medio ambientey cambio climático y contribución ala comunidad.

\section{MARCO TEÓRICO}

\subsection{Organizaciones del Tercer Sector y Responsabilidad Social Organizacional}

Las OTS son fundamentales en la dinámica social de un país, ya que son actores sociales que tienen como objetivo principal apoyar a sectores vulnerables dela ciudadanía. Tal como sostiene Donati(1997), las OTS pueden ser entendidas como "el producto de la diferenciación societal en condiciones de creciente complejidad social" (p. 116). Son estructuras organizacionales que operan en diferentes campos o ámbitos de la vida social: educación, ciudadanía, arte y cultura, asistencia social y desastres naturales, entre otras. El carácter altruista que las define, la "búsqueda del bien común" (Rubio, 2007, p. 277), hace que su meta principal sea transformar la realidad delos grupos vulnerables generando acciones orientadas a la generación de mejores oportunidades.

Las OTS se caracterizan, principalmente, por su autonomía, la auto-organización y la creación de redes relacionales estratégicas. Son entes separados del gobierno que requieren de voluntarios para el apoyo de sus actividades y de donantes para la captación de capital económico que permita llevar adelante las acciones previstas. En palabras de Ortiz (2019):

\footnotetext{
Se debe entender como Organización del Tercer Sector a todas aquellas que operan bajo fines nolucrativos, que buscan beneficiar a sectores vulnerablesy desprotegidos; y que cuentan con un número importante de voluntarios quienes apoyan principalmente con tiempo y conocimiento en el desarrollo de sus actividades, así como donantes que aportan capital económico y en especie (p. 99).
}

Debido a su carácter relacional, son organizaciones que deben interactuary fortalecer el vínculo con sus públicos de interés. Es decir, deben realizar acciones de RSO que les permitan generar lazos de confianza y obtener su apoyo y la comprensión para el logro de sus objetivos y para producir un impacto social en la comunidad donde se encuentran establecidas.

La RSO debe ser un esfuerzo de las OTS para desarrollar acciones éticas y congruentes con sus principales públicos: colaboradores, donantes, voluntarios, beneficiarios y comunidad. Es decir, llevar adelante una acción que les permita crear programas de diversa índole y tengan en cuenta, por ejemplo, el desarrollo de tareas que tengan que ver con el cuidado del medio ambiente 
y el cambio climático, la gestión de comportamientos éticos, contribuciones que promuevan el bienestar de la comunidad, prácticas de transparencia y buen gobierno, así como el respeto de cada uno de los públicos con quienes se involucra o interactúa.

El término RSO permite dejar de manifiesto que la organización es responsable ante todos sus grupos de interés y que buscará promover operaciones o acciones sustentables en el ámbito económico, social y ambiental (Rebeil \& Arevalo, 2017). Por lo tanto, el enfoque de la RSO que desarrollen las organizaciones debe tener una mirada integral: debe tener en cuenta el cuidado y la atención de los entornos y públicos con los que construye vínculos sociales. En palabras de Cajiga (2018):

El compromiso consciente y congruente de cumplir integralmente con la finalidad de la empresa, tanto en lo interno como en lo externo, considerando las expectativas económicas, sociales y ambientales de todos sus participantes, demostrando respeto por la gente, los valores éticos, la comunidad y el medio ambiente, contribuyendo así a la construcción del bien común (p. 4).

Estos principios rectores hacen que las OTS tengan presente que la RSO no debe quedar reducida a la realización de acciones sociales, sino que también las obliga a considerarse responsables por perseguir un fin social. "Atrás ha quedado el enfoque de responsabilidad social que se reducía en acciones principalmente filantrópicas o eran ajenas a un plan integral de RSO" (Rebeil y Ortiz, 2018, p. 290). Es por eso que deben crear códigos éticos y de conducta con sus públicos que resalten los valores de justicia, así como crear acciones que impacten de forma positiva en la comunidad donde se han establecido y no perjudique el cuidado y respeto del medio ambiente.

\subsection{Reputación en las OTS}

En la actualidad, el enfoque de reputación es un tema de interés para cualquier tipo de organización, dado que a través de su fortalecimiento pueden alcanzar reconocimiento y liderazgo frente a los públicos. De acuerdo con Villafañe (2004), la reputación debe entenderse como la armonía entre la identidad y la imagen consolidada en el tiempo. Es decir, una organización que decida fortalecer su reputación debe diseñar una identidad que le permita diferenciarse de las demás organizaciones y, además, construir y sostener una imagen coherente a lo largo del tiempo. Es importante que en la construcción de dicha identidad tomen en cuenta un plan de RSO, ya que es un factor fundamental en la percepción de la imagen con los públicos clave con los que interactúa.

La reputación en las OTS es un intangible estratégico que permea todos sus discursos y comportamientos. Por lo tanto, es esencial que las organizaciones construyan valores y atributos que les permitan establecer relaciones de confianza con sus públicos, ya que -en palabras de Arévalo y Ortiz (2019)- la 
reputación puede gestionarse a partir de las relaciones que las OTS generan con sus diferentes públicos de interés. Algunos de los beneficios que trae a las OTS la gestión de su reputación son: fortalecimiento de la confianza organizacional; atracción de fuentes de financiamiento -tanto de donantes individuales como de otras organizaciones-; atracción de capital social como voluntarios o donantes; fortalecimiento delas redes creadas con otras organizaciones. Los beneficios que la reputación le genera a las organizaciones es variado, pero para el éxito de esa gestión organizacional es fundamental que la comunicación se piense de manera estratégica y llegue adecuadamente a los públicos a través de diferentes herramientas de interacción.

\subsection{Comunicación publicitaria y medios digitales}

Es necesario subrayar quelas OTS son sistemas que necesitan comunicarse con sus públicos para funcionar de forma eficiente. Cada tipo de público tiene intereses ynecesidades distintas. Desdeelenfoquedela comunicación integralsedebenelegir lasdimensionesdela comunicación: creativa, mercadológica, corporativa, internay administrativa (Rebeil, Hidalgo \& Moreno, 2011), y cuál es la forma más oportuna para crear estrategias de comunicación que permitan la interacción.

Entendiendo que la RSO y la reputación deben ser comunicadas a todos los públicos, tanto internos como externos, esta investigación se centra en la comunicación publicitaria, y más específicamente: la comunicación publicitaria digital, la cual se encuentra dentro de la comunicación mercadológica. La comunicación publicitaria se dirige a públicos externos de las OTS: donantes, voluntarios, beneficiarios, comunidad, gobierno y medios de comunicación. Asimismo, debe estar alejada de una mera visión mercantil o comercial: el papel debe ser básicamente comunicativo, ya que sus decisiones "contribuyen a la construcción de conceptos como la solidaridad, el altruismo, la cooperación y la ayuda social en el marco de una ciudadanía cada vez más internacionalizada" (Rivas, 2005, p. 391).

Las OTS requieren hacer uso de la comunicación publicitaria a través de los medios tradicionales, pero la digitalización y la emergencia de nuevas formas de interacción son fundamentales para atraer voluntarios, captar donativos y fortalecer sus redes de vínculos con otras organizaciones. Frente a la nueva modernidad, los medios digitales se han convertido en una herramienta de bajo costo y con un alto alcance e impacto para comunicarse con todos los públicos. Esto invita a repensar los modelos de comunicación publicitaria bajo los entornos mediáticos actuales. Es decir, este tipo de organizaciones debe vislumbrar nuevas estrategias de comunicación publicitaria en medios y otros espacios digitales dado que:

En un contexto donde los consumidores y los ciudadanos pueden, a través de la comunicación digital, manifestar sus opiniones de campañas, productos, empresas y marcas de un modo constante, directo y crítico, no hay otra salida para la actividad publicitaria que evolucionar (Alvarado, 2012, p. 202). 
Porlotanto, unodelosprincipales retos quetienen estasorganizaciones escomprender los nuevos escenarios digitales, porque "las audiencias no se limitan sólo a recibir los mensajes, sino que también participan activamente en la elaboración de contenidos originales" (Kaufmann, 2014, p. 7). Esto significa que no sólo dependerá de la creatividad de los mensajes publicitarios, sino también de la creación de contenidos que aporten valor y resulten significativos para dichas audiencias. Asimismo, una exitosa comunicación publicitaria no sólo recae en la creatividad, el contenido o la correcta estrategia de medios, sino también en la veracidad, la ética y la transparencia de la realidad organizacional compartida en sus mensajes.

En síntesis, las OTS deben utilizar la comunicación publicitaria para dar a conocer sus acciones de RSO y fortalecer su reputación. A través de los nuevos entornos digitales pueden difundir sus objetivos sociales, sus logros, y sensibilizar a los públicos acerca de la causa social que persiguen. Esto les permitirá potenciar los vínculos y las redes creadas, lo que se traducirá en el aumento de las posibilidades de gestionar apoyo financiero, fortalecer el lazo creado con otras organizaciones y generar nuevos voluntarios.

\section{METODOLOGÍA}

\subsection{Objetivo}

Analizar y comparar -a través de un análisis de contenido- el nivel y el uso de la comunicación publicitaria digital que hacen 40 OTS de México, Chile, España e Inglaterra a través de sus páginas web con el propósito de dar a conocer las acciones de RSO de la organización. Por otro lado, dicho análisis de contenido permitirá entender cuáles organizaciones aprovechan de forma efectiva su página web institucional para comunicarse con sus públicos.

\subsubsection{Objetivos específicos}

A) Conocer cuál de las tres variables analizadas de la RSO utilizan principalmente las OTS de la muestra seleccionada.

в) Explicar de qué país de procedencia son las OTS que más utilizan la comunicación publicitaria digital para dar a conocer las acciones de RSO a sus públicos.

c) Describir los principales beneficios que aporta la comunicación publicitaria digital a las OTS.

\subsection{Hipótesis}

A mayor comunicación publicitaria digital de la RSO -mediante el uso y aprovechamiento que las OTS hacen de los medios y los nuevos espacios digitales-, existirá un mayor incremento de su reputación y de la confianza de sus principales públicos, lo que le permitirá alcanzar sus objetivos sociales: aumento de voluntarios, donantes y alianzas estratégicas. 


\subsection{Definición de muestra}

La muestra se obtuvo de la selección de 40 OTS de asistencia social, y de ella se seleccionaron 10 organizaciones de cada uno de los países analizados: de España se tomaron 10 organizaciones que están acreditadas por la Fundación Lealtad en España (Fundación Lealtad, 2018); de México se seleccionaron 10 acreditadas por Confío-Construyendo Organizaciones Civiles Transparentes, A.C. en México (Confío, 2018); de Chile fueron analizadas 10 organizaciones acreditadas por la Fundación Lealtad; finalmente, de Inglaterra se eligieron 10 organizaciones del directorio Charity Library (2018).

La selección de los países de la muestra se determinó por la presencia que tiene en ellos la Fundación Lealtad, una organización sin fines de lucro que ha desarrollado una metodología de análisis de transparencia y buenas prácticas para otorgar sello de acreditación a las OTS españolas, y también ha colaborado con la adaptación de esta metodología con la organización Confío en México y la Fundación Lealtad Chile. Se seleccionaron OTS que cuentan con el sello de acreditación de transparencia y buenas prácticas que es otorgado por alguno de estos tres organismos, quienes garantizan que son OTS que están realizando acciones de RSO y permite medir si dichas acciones se dan a conocer a través de su página web institucional haciendo uso de la comunicación publicitaria digital.

Las organizaciones que cuentan con dicho sello tienen presencia en México, Chile y España, otra de las razones por la cual se eligieron OTS de estos países. En el caso de Inglaterra, fue elegido dado que en el último tiempo ha presentado una evolución notoria en el desarrollo del Tercer Sector. Las organizaciones de Inglaterra seleccionadas también cuentan con el sello de transparencia y buenas prácticas otorgado por distintos organismos internacionales. Asimismo, la elección de las OTS de Inglaterra permite realizar un análisis comparativo entre dos países latinoamericanos y dos países europeos.

En cuanto ala decisión de elegir únicamente 10 organizaciones decada país, se determinó ese número de casos debido a que en el directorio dela Fundación Lealtad Chile sólo se encuentran acreditadas 10 OTS, y como el objetivo es realizar una comparación entre organizaciones con las mismas características, se dispuso trabajar con la misma cantidad de organizaciones que estuvieran acreditadas por la Fundación Lealtad y por Confío. De esta manera, y como en México, España e Inglaterra existía un mayor número de organizaciones acreditadas que en Chile, se utilizó el método de muestreo probabilístico aleatorio simple para elegir sólo a 10 organizaciones por cada país.

\subsection{Variables de investigación}

A la hora de medir la comunicación publicitaria digital de las acciones de RSO que las OTS seleccionadas realizan a través de sus páginas web institucionales, es decir, su sitio oficial, se decidió utilizar las variables propuestas por el 
DIRCOM(Asociación de Directivos de Comunicación), que es una asociación profesional que agrupa a los directivos y a los profesionales de la comunicación de las empresas, instituciones y consultoras (DIRCOM, 2019).

La herramienta metodológica de evaluación y autodiagnóstico creada por DIRCOM se organiza en base en cinco variables, cuyo peso es ponderado según la relevancia para una gestión estratégica de la RSC, alineada con la generación de valor reputacional. Las variables son: comportamiento ético; transparencia y buen gobierno; comportamiento con los empleados; medio ambiente y cambio climático; y, finalmente, contribución a la comunidad. Pero es necesario aclarar que en el marco de esta investigación se eligieron únicamente tres de las cinco variables antes mencionadas: comportamiento ético, medio ambiente y cambio climático y la variable contribución a la comunidad; cada una con sus propios indicadores para la medición.

Asimismo, es pertinente aclarar que la utilización de esta metodología se sostiene o se decidió utilizarse porque DIRCOM la creó tomando como premisa fundamental qué tanto la reputación como la responsabilidad social corporativa no deben ser considerados como activos separados, sin influencia mutua, ya que se considera que la gestión corporativa que aporta mayor valor es la que integra ambos aspectos. En ese marco, la herramienta de evaluación y autodiagnóstico permite obtener una valoración final en la que se detectan áreas clave para trabajar y se ofrecen una serie de recomendaciones de mejora para optimizar la gestión de la RSC y generar mayor valor reputacional (DIRCOM, 2019).

\subsection{Diseño de investigación}

Esta investigación es de tipo cuantitativo y se realizó mediante la técnica de análisis de contenido. Se analizaron las páginas web oficiales de las OTS elegidas en la muestra con el fin de medir su RSO. Sólo se analizaron las páginas web con el contenido que tenían publicado hasta el día 30 de junio de 2019. Las etapas de la investigación fueron: 1) elección de variables e indicadores; 2) definición de la muestra; 3 ) revisión y análisis de las páginas web de las OTS; 4) interpretación de los resultados.

\section{RESULTADOS}

\subsection{Comparativo de comunicación publicitaria digital de RSO por variable}

Como se ha mencionado, es importante que las OTS utilicen la comunicación publicitaria digital para dar a conocer sus acciones de RSO a sus públicos por medio de su página web. Bajo esta óptica se midió si las organizaciones seleccionadas para este estudio utilizan dicho medio de comunicación digital 
tomando en cuenta las tres variables antes mencionadas: comportamiento ético, medio ambiente y cambio climático y contribución a la comunidad.

Para medir estas variables se utilizaron los indicadores de la herramienta DIRCOM con sus propias ponderaciones, ya que es un instrumento internacional validado. El análisis se elaboró en dos etapas: en la primera, se revisaron las páginas web oficiales delas OTS teniendo en cuenta si los contenidos publicados mostraban información con respecto a los indicadores trabajados. La información recabada para cada variable se codificó en una tabla que incluía dos opciones para cada indicador: Sí cumple y No cumple.

En la segunda etapa, se midió el nivel de comunicación publicitaria digital de las OTS por país. Para evaluar el total de organizaciones se diseñó una serie de intervalos que permitieron ponderar los porcentajes en una escala entre $0 \%$ y $100 \%$, lo cual permitió determinar el total del porcentaje de OTS que utilizan la comunicación publicitaria digital (Tabla 1) y observar los principales retos que tienen en lo referido a esa cuestión.

Tabla 1. Nivel de comunicación publicitaria digital de las OTS

\begin{tabular}{|c|c|c|}
\hline $\begin{array}{c}\text { Intervalo de } \\
\text { porcentaje de } \\
\text { organizaciones }\end{array}$ & $\begin{array}{c}\text { Nivel de } \\
\text { comunicación } \\
\text { publicitario digital } \\
\text { de la RSO }\end{array}$ & Significado \\
\hline $\begin{array}{c}0 \%-20 \% \\
\text { organizaciones }\end{array}$ & Precario & $\begin{array}{l}\text { Significa que un mínimo porcentaje de } \\
\text { organizaciones utilizan la comunicación publicitaria } \\
\text { digital para dar a conocer sus acciones de } \\
\text { responsabilidad social organizacional, por lo tanto, } \\
\text { es precario el avance de la comunicación publicitaria } \\
\text { de la RSO en ese país. }\end{array}$ \\
\hline $\begin{array}{c}21 \%-40 \% \\
\text { organizaciones }\end{array}$ & Bajo & $\begin{array}{l}\text { Significa que un bajo porcentaje de organizaciones } \\
\text { utilizan la comunicación publicitaria digital para } \\
\text { dar a conocer sus acciones de responsabilidad social } \\
\text { organizacional, por lo tanto, es bajo el avance de la } \\
\text { comunicación publicitaria de la RSO en ese país. }\end{array}$ \\
\hline $\begin{array}{c}41 \%-60 \% \\
\text { organizaciones }\end{array}$ & Regular & $\begin{array}{l}\text { Significa que un porcentaje medio de organizaciones } \\
\text { utilizan la comunicación publicitaria digital para } \\
\text { dar a conocer sus acciones de responsabilidad social } \\
\text { organizacional, por lo tanto, es regular el avance de } \\
\text { la comunicación publicitaria de la RSO en ese país. }\end{array}$ \\
\hline $\begin{array}{c}61 \%-80 \% \\
\text { organizaciones }\end{array}$ & Aceptable & $\begin{array}{l}\text { Significa que un porcentaje significativo de } \\
\text { organizaciones utilizan la comunicación } \\
\text { publicitaria digital para dar a conocer sus acciones } \\
\text { de responsabilidad social organizacional, por lo } \\
\text { tanto, es aceptable el avance de la comunicación } \\
\text { publicitaria de la RSO en ese país. }\end{array}$ \\
\hline $\begin{array}{c}81 \%-100 \% \\
\text { organizaciones }\end{array}$ & Ideal & $\begin{array}{l}\text { Significa que un alto porcentaje de organizaciones } \\
\text { utilizan la comunicación publicitaria digital para } \\
\text { dar a conocer sus acciones de responsabilidad social } \\
\text { organizacional, por lo tanto, es ideal el avance de la } \\
\text { comunicación publicitaria de la RSO en ese país. }\end{array}$ \\
\hline
\end{tabular}

Fuente: elaboración propia. 


\subsubsection{Comportamiento ético}

Como ya se mencionó, uno de los principales retos que tienen las OTS es construir confianza y reputación con sus públicos clave. Esto implica una mayor exigencia para comunicar sus acciones y mostrar el despliegue de comportamientos éticos y transparentes. Para lograrlo las OTS deben diseñar códigos éticos que guíen sus conductas y los comportamientos diarios de todos los integrantes de la organización, involucrando tanto el funcionamiento interno como la relación que se establece con el exterior de la misma.

Para medir la variable de comportamiento ético, en una primera fase se analizaron los ocho indicadores que se muestran en la Tabla 2, con el fin de identificar si las OTS utilizaban la comunicación publicitaria digital para dar a conocer sus acciones de RSO enfocadas al comportamiento ético. Para esto se utilizaron las opciones: Sí cumple y No cumple, por país. Los resultados se muestran a continuación:

Tabla 2. Comparativo de indicadores de comportamiento ético

\begin{tabular}{|c|c|c|c|c|c|}
\hline $\begin{array}{c}\text { Indicadores de } \\
\text { comportamiento ético }\end{array}$ & México & España & Chile & Inglaterra & $\begin{array}{c}\text { Promedio } \\
\text { (países) }\end{array}$ \\
\hline $\begin{array}{l}\text { a) Código ético o de } \\
\text { conducta }\end{array}$ & $0 \%$ & $30 \%$ & $40 \%$ & $60 \%$ & $33 \%$ \\
\hline $\begin{array}{l}\text { b) Código ético para } \\
\text { proveedores }\end{array}$ & $14 \%$ & $20 \%$ & $18 \%$ & $36 \%$ & $22 \%$ \\
\hline $\begin{array}{l}\text { c) Canales de } \\
\text { comunicación } \\
\text { para gestionar el } \\
\text { comportamiento ético }\end{array}$ & $22 \%$ & $80 \%$ & $50 \%$ & $70 \%$ & $56 \%$ \\
\hline $\begin{array}{l}\text { e) Comité o comisión } \\
\text { de ética }\end{array}$ & $0 \%$ & $50 \%$ & $40 \%$ & $40 \%$ & $33 \%$ \\
\hline $\begin{array}{l}\text { f) Política de } \\
\text { anticorrupción }\end{array}$ & $0 \%$ & $20 \%$ & $20 \%$ & $50 \%$ & $23 \%$ \\
\hline $\begin{array}{l}\text { g) Protocolo de } \\
\text { actuación frente a } \\
\text { conflictos éticos }\end{array}$ & $10 \%$ & $0 \%$ & $20 \%$ & $20 \%$ & $12 . \%$ \\
\hline $\begin{array}{l}\text { h) Herramientas } \\
\text { para preservarla } \\
\text { privacidad de donantes } \\
\text { y beneficiarios }\end{array}$ & $30 \%$ & $80 \%$ & $30 \%$ & $80 \%$ & $55 \%$ \\
\hline $\begin{array}{l}\text { i) Adhesión a iniciativas } \\
\text { que impulsen el } \\
\text { comportamiento ético }\end{array}$ & $40 \%$ & $80 \%$ & $40 \%$ & $80 \%$ & $60 \%$ \\
\hline $\begin{array}{l}\text { Resultado de } \\
\text { comportamiento ético } \\
\text { por país }\end{array}$ & $15 \%$ & $45 \%$ & $32 \%$ & $54 \%$ & $37 \%$ \\
\hline
\end{tabular}

Fuente: elaboración propia. 
Para construir una conducta ética se debe establecer un código de normas y valores que guíe los comportamientos e interacciones con sus públicos de una forma congruente, ya que como plantea Olmos (2007): "si usted quiere una organización exitosa a largo plazo, debe haber congruencia entre lo que dice y sus actividades diarias" (p. 87). Es decir, las acciones y las decisiones adoptadas deben estar alineadas con los discursos y mensajes rectores de la organización.

Con respecto al indicador "código ético o de conducta" se observa que: el $60 \%$ de las OTS de Inglaterra cuenta con este tipo de códigos, seguido de Chile con un 40\% y España que se aproxima al 30\%; mientras que en México ninguna de las organizaciones analizadas cuenta con este tipo de códigos. Esto significa que, en promedio, de las 40 organizaciones analizadas sólo un 33\% comunica en su página web institucional su código ético y de conducta. Con respecto al indicador de "código de conducta para proveedores": $36 \%$ de las organizaciones de Inglaterra sí cuenta con él, seguido por España con un 20\%, Chile con un $18 \%$ y finalmente México con el 14\%. En promedio, sólo el 22\% de las organizaciones analizadas lo publica.

En cuanto a los "canales de comunicación para gestionar el comportamiento ético", se revisó que dentro de la página web institucional de las OTS existieran vías de comunicación específicas para que los públicos clave puedan canalizar denuncias, dudas o incidencias acerca del comportamiento de cualquier integrante de la organización. Se encontró que el $80 \%$ de las organizaciones españolas sí cuenta con estas vías, seguido por el 70\% de las organizaciones de Inglaterra, el 50\% en Chile y 22\% en México. En promedio, el 56\% de las organizaciones analizadas cuenta con canales de comunicación para gestionar el comportamiento ético.

Con respecto al indicador que señala la existencia de un "comité o comisión de ética", se revisó que las organizaciones dentro de su página web publicaran información sobre quienes deben ser los encargados de vigilar el cumplimiento del código de ética y de conducta antes mencionado. Los resultados encontrados fueron que el 50\% de las OTS españolas sí cuenta con un comité o comisión de ética, seguido por las organizaciones de Chile e Inglaterra con un $40 \%$, mientras que en las OTS mexicanas analizadas no existen publicaciones acerca de este mecanismo fiscalizador. En promedio, el $33 \%$ de las organizaciones comunica en su página web información acerca de su comité o comisión de ética.

Para el indicador "política de anticorrupción", que incluye los principios que definen cómo deben conducirse o actuar los integrantes dela organización frente a determinadas situaciones, se encontró que el $50 \%$ delas OTS de Inglaterracuenta con este tipo de políticas, seguido por las organizaciones de Españay de Chile con un 20\%, mientras que en México ninguna organización analizada 
lo tiene publicado. En total sólo el 23\% de las organizaciones analizadas tiene publicadas en su página web políticas de anticorrupción.

Para el indicador "protocolos de actuación frente a conflictos éticos" se observó que las organizaciones publicaran información acerca del proceso que se debe seguir en caso de originarse problemas que involucren o tengan que ver con la organización. Se observó que el $20 \%$ de las organizaciones de Inglaterra y de Chile y el 10\% de las organizaciones mexicanas cuentan con un protocolo de este tipo, mientras que las españolas no tienen publicados protocolos que guíen su actuar frente a conflictos éticos. El promedio de organizaciones que describen su protocolo de actuación en su página web es únicamente el $12 \%$.

Otro indicador de suma importancia para este tipo de organizaciones fue el análisis de las "herramientas para preservar la privacidad de los donantes y beneficiarios”. Para esto se buscó determinar si las organizaciones tenían publicadas políticas que garantizaran la privacidad de sus donantes y beneficiarios. Se observó que las organizaciones de Inglaterra y de España son las que más las publican (80\%), mientras que en los casos de México y de Chile llega en cada caso a un $30 \%$. En promedio, el $55 \%$ de las organizaciones analizadas comunica sus políticas de privacidad.

Finalmente, se revisó si las organizaciones comunicaban "iniciativas que impulsen el comportamiento ético” tanto dela organización como de sus públicos, y se obtuvo que el $80 \%$ de las organizaciones de España y de Inglaterra sílo hacen, mientras que en los casos de México y Chile tan sólo un $40 \%$ comunica o adhiere a este tipo de iniciativas. En suma, el $60 \%$ publica iniciativas para impulsar su comportamiento ético.

\subsubsection{Medio ambiente y cambio climático}

Las acciones que realizan las OTS enfocadas al cuidado y gestión del medio ambiente y el cambio climático les demuestra a sus públicos el compromiso no sólo con su causa social, sino también con los entornos con los que interactúan. Es importante mencionar que todas aquellas organizaciones preocupadas por la problemática medioambiental tienen garantizada una sólida reputación, pero constantemente deben analizar que las acciones que llevan a cabo no sean afectadas o incumplan los objetivos propuestos.

Para medir la variable medio ambiente y cambio climático se analizaron, en una primera fase, nueve indicadores con el fin de identificar si las OTS utilizaban la comunicación publicitaria digital para dar a conocer sus acciones de RSO enfocadas en la temática medioambiental. Para esto se utilizaron las opciones: Sí cumple y No cumple, por país. Los resultados se muestran a continuación en la Tabla 3. 
Tabla 3. Indicadores de medio ambiente y cambio climático

\begin{tabular}{|l|c|c|c|c|c|}
\hline $\begin{array}{l}\text { Indicadores de medio ambiente y } \\
\text { cambio climático }\end{array}$ & México & España & Chile & Inglaterra & Promedio \\
\hline $\begin{array}{l}\text { a) Medición y gestión del impacto } \\
\text { ambiental }\end{array}$ & $0 \%$ & $0 \%$ & $40 \%$ & $20 \%$ & $15 \%$ \\
\hline $\begin{array}{l}\text { b) Proyectos para la reducción de } \\
\text { consumo de recursos naturales o } \\
\text { fomento de energías renovables. }\end{array}$ & $0 \%$ & $0 \%$ & $40 \%$ & $20 \%$ & $15 \%$ \\
\hline $\begin{array}{l}\text { c) Procedimiento de gestión/compra } \\
\text { de productos }\end{array}$ & $0 \%$ & $0 \%$ & $20 \%$ & $10 \%$ & $7.5 \%$ \\
\hline d) Informe medioambiental & $0 \%$ & $0 \%$ & $10 \%$ & $20 \%$ & $7.5 \%$ \\
\hline $\begin{array}{l}\text { e) Iniciativas para mitigar los impactos } \\
\text { medioambientales }\end{array}$ & $0 \%$ & $0 \%$ & $30 \%$ & $20 \%$ & $12.5 \%$ \\
\hline f) Programas de reciclaje & $0 \%$ & $20 \%$ & $20 \%$ & $10 \%$ & $12.5 \%$ \\
\hline $\begin{array}{l}\text { g) Posicionamiento frente al cambio } \\
\text { climático }\end{array}$ & $0 \%$ & $0 \%$ & $10 \%$ & $10 \%$ & $5 \%$ \\
\hline h) Certificaciones medioambientales & $0 \%$ & $0 \%$ & $20 \%$ & $10 \%$ & $7.5 \%$ \\
\hline $\begin{array}{l}\text { i) Adhesiones a Iniciativas } \\
\text { medioambientales }\end{array}$ & $0 \%$ & $0 \%$ & $40 \%$ & $20 \%$ & $15 \%$ \\
\hline $\begin{array}{l}\text { Resultado de medio ambientey } \\
\text { cambio climático por país }\end{array}$ & $\mathbf{0 \%}$ & $\mathbf{2 \%}$ & $\mathbf{2 5 \%}$ & $\mathbf{1 5 \%}$ & $\mathbf{1 0 \%}$ \\
\hline
\end{tabular}

Fuente: elaboración propia.

Para esta investigación se observó que las organizaciones de México no publican en su página web institucional acciones con relación a la variable medio ambiente y cambio climático, mientras que en España sólo el 20\% de las organizaciones analizadas comparte en su página web "programas de reciclaje", centrados en mitigar la problemática medioambiental. Con respecto a las organizaciones de Chile, las acciones que mayormente se realizan (el 40\% de las organizaciones) tiene que ver con indicadores ligados a la "medición y la gestión del impacto ambiental”, la realización de "proyectos para reducir el consumo de recursos naturales o el fomento de energías renovables" yla "adhesión a iniciativas medioambientales". Mientras que un $30 \%$ de las organizaciones chilenas publican "iniciativas para mitigar los impactos medioambientales", un $20 \%$ dice contar con "procedimientos de gestión y compra de productos", "programas de reciclaje" y "certificaciones medioambientales", y sólo el 10\% publica un "informe medioambiental" ysu "postura frente al cambio climático".

Con respecto a la muestra de Inglaterra, el $20 \%$ de las organizaciones analizadas comparten acciones enfocadas en la "medición y gestión del impacto ambiental", "proyectos parala reducción de consumo de recursos naturales", informes y adhesiones e iniciativas para mitigar los impactos medioambientales. Sólo un 10\% comunica "procedimientos de gestión de compra de productos", "programas de reciclaje", "posicionamiento frente al cambio climático" y "certificaciones medioambientales". 
Estos resultados permitieron observar que esta variable -medio ambiente y cambio climático - tiene una baja implementación por parte de las OTS de los cuatro países analizados, ya que los promedios por indicador son los siguientes: "medición y gestión del impacto ambiental" (15\%), "proyectos parala reducción de consumo de recursos naturales o fomento de energías renovables" (15\%), "procedimiento de gestión/compra de productos" (7.5\%), "informe medioambiental" (7.5\%), "iniciativas para mitigar los impactos medioambientales" (12.5\%), "programas de reciclaje" (12.5\%), "posicionamiento frente al cambio climático" (5\%), "certificaciones medioambientales" (7.5\%) y "adhesiones a iniciativas medioambientales" (15\%).

\subsubsection{Contribución a la comunidad}

La contribución ala comunidad implica realizar acciones que generen impacto social; es decir, que beneficien no sólo a los principales públicos de la organización, sino a la comunidad donde se encuentran establecidas. Esta variable va más allá del cumplimiento del fin social que persiguen las OTS: significa que deben realizar acciones que cambien la realidad delos públicos con los que se relacionan y de las personas de la localidad a través de una gestión sostenible a largo plazo.

Para medir dicha variable se tuvieron en cuenta cuatro indicadores con el fin de identificar si las OTS utilizaban la comunicación publicitaria digital para dar a conocer sus acciones de RSO enfocadas en la contribución a la comunidad. Para esto se utilizaron las opciones: Sí cumple y No cumple, por país. Los resultados se muestran a continuación en la Tabla 4.

Tabla 4. Indicadores de contribución a la comunidad

\begin{tabular}{|l|c|c|c|c|c|}
\hline $\begin{array}{c}\text { Indicadores de contribución } \\
\text { a la comunidad }\end{array}$ & México & España & Chile & Inglaterra & Promedio \\
\hline $\begin{array}{l}\text { a) Política de relaciones con la } \\
\text { comunidad }\end{array}$ & $0 \%$ & $100 \%$ & $90 \%$ & $50 \%$ & $60 \%$ \\
\hline $\begin{array}{l}\text { b) Sistema de evaluación del } \\
\text { impacto social }\end{array}$ & $10 \%$ & $50 \%$ & $50 \%$ & $80 \%$ & $48 \%$ \\
\hline $\begin{array}{l}\text { c) Programas de integración } \\
\text { social }\end{array}$ & $70 \%$ & $90 \%$ & $90 \%$ & $100 \%$ & $88 \%$ \\
\hline $\begin{array}{l}\text { d) Sistema de medición del } \\
\text { desempeño }\end{array}$ & $10 \%$ & $60 \%$ & $70 \%$ & $70 \%$ & $53 \%$ \\
\hline $\begin{array}{l}\text { Resultado de contribución a } \\
\text { la comunidad por país }\end{array}$ & $\mathbf{2 2 \%}$ & $\mathbf{7 5 \%}$ & $\mathbf{7 5 \%}$ & $\mathbf{7 5 \%}$ & $\mathbf{6 2} \%$ \\
\hline
\end{tabular}

Fuente: elaboración propia.

Los resultados para esta variable por indicador fueron los siguientes: en lo referido a "política de relaciones con la comunidad", el 100\% de las OTS de España muestran referencias y comunicaciones ligadas al vínculo comunitario, mientras que las organizaciones de Chile lo hacen en un 90\%, le siguen las de Inglaterra con el 50\% y finalmente las OTS de México, que aún no han abor- 
dado o comunicado dicha política. En promedio, el 60\% de las organizaciones analizadas comunica políticas de relaciones con la comunidad.

En cuanto al indicador "sistema de evaluación del impacto social", las organizaciones de Inglaterra son las que más lo han implementado (80\%), seguido de Chile e Inglaterra (50\%) y finalmente las organizaciones de México (10\%). Con respecto a los “programas de integración social”, todas las organizaciones analizadas de Inglaterra comunican ese tipo de programas, las españolas y las chilenas lo hacen en un alto porcentaje (90\%) y las mexicanas rondan el 70\%. Finalmente, para el "sistema de medición del impacto" se obtuvo que el 70\% de las organizaciones de Chile e Inglaterra sí evalúan sus acciones, seguidas de las organizaciones españolas (60\%) y por último México, con apenas el $10 \%$ de las organizaciones analizadas que comunican tener un sistema de medición de impacto.

\subsection{Comparativo de comunicación publicitaria digital de RSO por variable de cada país}

Para explicar con claridad cuáles acciones de RSO dan a conocer las OTS a través de su comunicación publicitaria digital, en el Gráfico 1 se presenta el comparativo del resultado total de las variables: comportamiento ético, medio ambiente y cambio climático y contribución a la comunidad. Dicho comparativo se obtuvo sumando el porcentaje del total de cada uno de los indicadores de cada variable por país y, posteriormente, se dividió entre el número de los indicadores tenidos en cuenta.

Gráfico 1

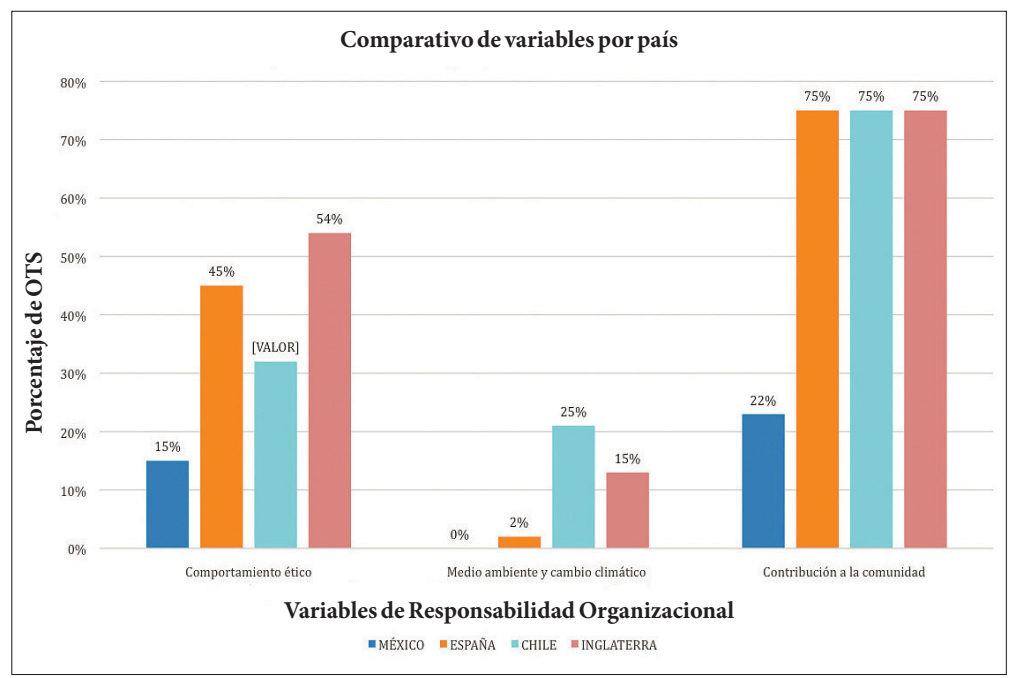

Fuente: elaboración propia. 
Para el comportamiento ético se sumaron los ocho porcentajes obtenidos por cada país y luego se dividió entre los ocho indicadores medidos en esta variable. En los resultados finales se observó que el país que más comunica su comportamiento ético es Inglaterra (54\%), seguido por las organizaciones de España (45\%), Chile (32\%) y México (15\%). Si bien es cierto que en Inglaterra cumplen más con este tipo de acciones, hay que tener en cuenta que solamente representa a la mitad de las organizaciones analizadas. Esto significa que los cuatro países deben trabajar en desarrollar acciones que fortalezcan las acciones y la comunicación que tengan que ver con la variable comportamiento ético.

Con respecto a la variable medio ambiente y cambio climático, se sumaron los porcentajes obtenidos por cada país y, posteriormente, fue dividido teniendo en cuenta los nueve indicadores medidos en relación a esta variable. En los resultados se observó que las OTS que más comunican sus acciones de cuidado del medio ambientey el cambio climático son de Chile(25\%), seguidas por las organizaciones de Inglaterra (15\%) y de España con tan sólo un 2\%; mientras que las organizaciones de México no publican acciones acerca de esta variable. En general, se puede observar que, de las tres variables analizadas, esta es la que menos desarrollan y comunican las organizaciones analizadas, sin importar el país de procedencia. Esto sucede porque las OTS tienen la idea de que al perseguir un objetivo social ya estarían respondiendo al llamado de las organizaciones socialmente responsables. Sin embargo, como se mencionó, la actividad de las OTS implica un acto voluntario con todos sus entornos, y un aspecto central es la disposición de alternativa o la adhesión con mecanismos de cuidado y protección del medio ambiente.

Finalmente, para la variable contribución a la comunidad se sumaron los cuatro porcentajes obtenidos por país y se procedió a dividirlo entre los cuatro indicadores medidos. En los resultados se observó quelas organizaciones delos países que más comunican sus acciones de contribución a la comunidad fueron las de Chile, España e Inglaterra, con el 75\%; finalmente las OTS de México con el $22 \%$. Como son organizaciones que tienen un fin social, se observa que las acciones que más han implementado y que comunican son aquellas que se enfocan a la contribución a la comunidad.

En síntesis, se observó que la variable de RSO que más dan a conocer las 40 organizaciones analizadas a través de la comunicación publicitaria digital es: contribución a la comunidad (62\%). Le siguen comportamiento ético (37\%) y cuidado del medio ambiente y cambio climático (14\%). Esto significa que este tipo de organizaciones, al perseguir un fin social y estar preocupadas por contribuir en la ayuda de diferentes sectores vulnerables, realizan y comunican acciones que tienen que ver con el intento de mejorar la calidad de vida de los ciudadanos de las poblaciones en las que se encuentran establecidas. Además, están empezando agenerar esfuerzos para compartir su comportamientoético. 
No obstante, los datos arrojados por la investigación permiten deducir que aún no están creando y comunicando acciones que beneficien o tengan en cuenta de manera específica la cuestión medioambiental.

\subsection{Comparativo de comunicación publicitaria digital de RSO por país}

Después de presentar el comparativo de los resultados obtenidos por cada una de las tres variables de medición de la RSO, en el Gráfico 2 se presenta el porcentaje total de las organizaciones que componen la muestra.

Gráfico 2

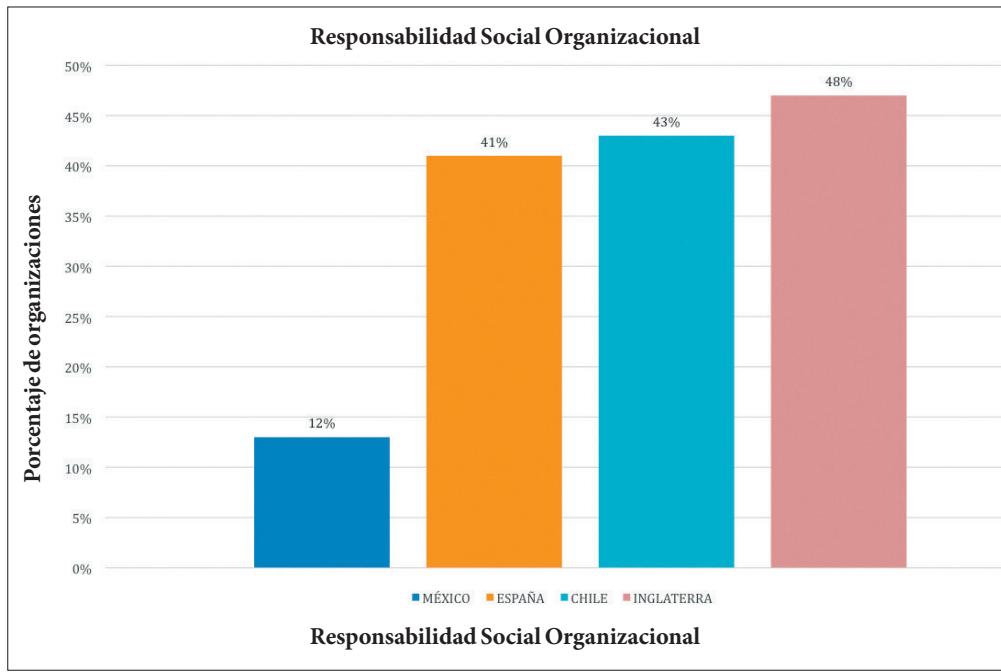

Fuente: elaboración propia.

Para obtener el total de cada uno de los países (o, mejor dicho, de las OTS de los países tomados para la muestra), se procedió a sumar el porcentaje obtenido de las tres variables analizadas de cada país y se dividió por el número de variables. Los resultados que se obtuvieron fueron que las organizaciones que más utilizan la comunicación publicitaria digital para dar a conocer sus acciones de RSO en sus páginas web son de los siguientes países: Inglaterra (48\%), Chile (43\%), España (41\%) y finalmente México (12\%).

De acuerdo con el porcentaje total de las organizaciones, se puede determinar lo siguiente: con respecto a los casos de Inglaterra, Chile y España, el total de OTS analizadas se encuentra en un nivel regular: esto significa que un porcentaje medio de las organizaciones utiliza la comunicación publicitaria digital para dar a conocer sus acciones de RSO. En tal sentido, el avance de la 
comunicación publicitaria digital de la RSO presenta retos importantes, ya que es utilizada por menos del $50 \%$ de las organizaciones analizadas. Con respecto a México, un mínimo porcentaje de las organizaciones se vale de la comunicación publicitaria digital para dar a conocer sus acciones, demostrando un precario avance en el uso de las páginas web institucionales y los medios digitales como herramienta de interacción comunicativa y promoción de las acciones ligadas a las variables analizadas.

Si bien es cierto que las OTS de México se encuentran muy por debajo de las organizaciones de Chile, España e Inglaterra analizadas, más de la mitad de las organizaciones de estos tres países aún no han desarrollado y comunicado acciones de RSO a través de las páginas web institucionales. Esto significa que las OTS de los cuatro países presentan retos importantes para redefinir y orientar una comunicación estratégica de la RSO que tenga en cuenta los aportes de la comunicación publicitaria digital. Para enfrentarlos, deben crear acciones integrales y hacer partícipes a sus públicos -entre otras formas- a través de la comunicación publicitaria digital, lo cual permitirá fortalecer la reputación y la confianza y afianzar los mecanismos ligados a variables como el comportamiento ético, las temáticas ligadas al medio ambiente y el cambio climático y, de un modo más general, las acciones que contribuyan a la comunidad.

\section{CONCLUSIONES Y PRINCIPALES HALLAZGOS}

Los resultados arrojados en este estudio empírico permitieron dar respuesta a los objetivos planteados en esta investigación: A) Conocer cuál de las tres variables analizadas de la RSO utilizan las OTS de la muestra analizada: se observa que, de las tres variables analizadas, la que más comunican a través de su página web institucional tiene que ver con la contribución a la comunidad, seguida de la variable comportamiento ético, y finalmente cuidado del medio ambiente y cambio climático.

La variable contribución a la comunidad se posiciona en primer lugar, esencialmente, porque al tratarse de organizaciones con fines sociales tienen como meta principal contribuir a la comunidad a través de sus acciones y los servicios brindados. Por esta razón, utilizan la comunicación publicitaria digital principalmente para dar a conocer sus acciones de RSO que han implementado para sus beneficiarios, voluntarios y donantes.

Se vislumbran algunos esfuerzos por comunicar sus comportamientos éticos; no obstante, las OTS analizadas tienen por delante mucho camino por recorrer. Finalmente, la variable a la que deberían otorgarle un mayor esfuerzo organizacional es: cuidado del medio ambiente y cambio climático. En la actualidad es importante que todas las OTS, sin importar si su objetivo social se encuentra centrado en la problemática o el entorno medioambiental, diseñen acciones que ayuden a mitigar el daño que sufre el planeta. Toda 
OTS que diseñe estrategias de RSO debe hacer tener una mirada integral, no sólo pensando para su entorno organizacional. Es decir, tiene que tener la capacidad de influir de forma positiva en los entornos con las que interactúa e interpelar a las personas u organizaciones con el propósito de generar acciones conjuntas que valoricen temas de interés común.

Con respecto al objetivo B), explicar de qué país procedencia son las OTS que más utilizan la comunicación publicitaria digital para dar a conocer sus acciones de RSO con sus públicos, la investigación puso de manifiesto que las OTS de los cuatro países analizados deben fortalecer sus acciones de RSO y diseñar una estrategia en torno de la comunicación publicitaria digital. Si bien es cierto que los datos obtenidos colocan a las organizaciones de Inglaterra en primer lugar, es menos de la mitad de sus organizaciones las que realizan acciones de RSO y las comunican a sus públicos en medios digitales. Con respecto a los casos de España y Chile, apenas el 40\% de las organizaciones analizadas comunica en su página web institucional sus acciones de RSO. Esto significa que las organizaciones analizadas de estos tres países se encuentran en un nivel regulary, por lo tanto, deben tener presentes los retos que implica el uso responsable de las nuevas herramientas de comunicación publicitaria digital.

Llama la atención los bajos resultados obtenidos por las OTS mexicanas: sólo un porcentaje mínimo de éstas utiliza la comunicación publicitaria digital para informar de las acciones que tengan que ver con la RSO. Estos resultados permiten exponer que la mayoría de las OTS mexicanas analizadas deben realizar un gran esfuerzo para comunicar sus acciones de RSO, rasgo que las diferencia de las OTS de Inglaterra, España y Chile. Además, se hace visible que las OTS con presencia en el continente europeo tienen un mayor avance en materia de comunicación publicitaria digital quelas OTSlatinoamericanas analizadas.

En general, los resultados obtenidos permiten revelar a las OTS de los cuatro países que existen áreas de oportunidad en el campo de la comunicación publicitaria, la RSOy el uso delos medios digitales yla página web institucional. Para hacer frente a esos retos es necesario que las organizaciones de este tipo cambien su percepción frente a la RSO y entender que no es exclusiva de organizaciones privadas o de las organizaciones que manejan grandes presupuestos. Asimismo, estas organizaciones deben conocer los principales beneficios que trae el uso de la comunicación publicitaria digital de la RSO. Finalmente, para resolver algunos de los desafíos mencionados deben crear estrategias integrales que fortalezcan su comportamiento ético, permitan abordar temas relacionados con el medio ambiente y cambio climático, contribuyan al desarrollo comunitario y, en lo estrictamente comunicacional, ofrezcan instancias de intercambio que haga participe a sus públicos a través de la comunicación publicitaria digital. 
En cuanto al objetivo C) Describir los principales beneficios que aporta la comunicación publicitaria digital a las OTS, es importante mencionar que las organizaciones analizadas presentan un gran avance en materia de acciones de RSO y, como ya se mencionó, son organizaciones con sello de transparencia y buenas prácticas. Es decir, son estructuras organizacionales que se han preocupado por implementar acciones de RSO. Sin embargo, no están aprovechando sus medios digitales para dar a conocer a sus públicos clave todas las acciones que realizan. Si bien es cierto que para que las organizaciones tengan un mejor desempeño organizacional requieren un cambio en sus subsistemas y comportamientos internos, la ayuda de la comunicación publicitaria digital permitirá fortalecer su reputación con sus públicos y la red de vínculos con los que interactúa.

Además, es fundamental que comprendan los beneficios y la importancia que tienen la RSO y la comunicación publicitara digital en la interacción con sus públicos y en el éxito de los objetivos organizacionales. Un adecuado plan integral de RSO con apoyo de la comunicación publicitaria digital contribuye a sensibilizar a sus públicos, fortalece el carácter relacional atrayendo talento humano como colaboradores y voluntarios, y también puede contribuir a ganar la confianza de sus donantes para que incrementen sus aportes.

Finalmente, para dar respuesta al objetivo general, se concluye que estas organizaciones no están comunicando a sus públicos sus acciones de RSO de manera integral. Es decir que necesitan implementar la comunicación publicitaria y aprovechar las herramientas digitales para establecer diálogos estratégicos con sus públicos, para queles permitan fortalecer su reputación organizacional y obtener así el apoyo y la solidaridad de sus públicos; pieza fundamental para alcanzar los objetivos organizacionales propuestos.

\section{REFERENCIAS}

Aguilar, V. (2006). Las organizaciones de la sociedad civil en México: su evolución y principales retos (tesis doctoral). México: Universidad Iberoamericana.

Alvarado, L. M. (2012). La publicidad en el marco dela comunicación para el desarrollo: hacia un nuevo modelo de publicidad. Cuadernos de Informacióny Comunicación, 17, 191-207. DOI: http://dx.doi.org/10.5209/rev_CIYC.2012.v17.39264

Arévalo, M. R. \& Ortiz, R. H. (2019). Relaciones públicas, reputación y responsabilidad social. En M. A. Rebeil, R. Arévalo \& P. Durán, Comunicación y Teconología, potenciar las organizaciones (pp. 69-88). México: BUAP.

Cajiga, C. J. (2018). El concepto de Responsabilidad Social Empresarial. Recuperado de: https://www.cemefi.org/esr/images/stories/pdf/esr/concepto_esr.pdf 
Donati, P. (1997). El desarrollo de las Organizaciones del Tercer Sector en el proceso de modernizacion y más allá. Reis (79), 113-141. DOI: 10.2307/40184010

Kaufmann, A. J. (2014). De la publicidad tradicional a la publicidad digital. Recuperado de: https://www.researchgate.net/publication/267508677

Ortiz, R. H. (2019). La comunicación integral en las Organizaciones del Tercer Sector Sintaxis. Revista del Centro de Investigación para la Comunicació Aplicada, 1(2), 95-112. Recuperado de:http://pegaso.anahuac.mx/accesoabierto/publicaciones. php.

Olmos, A. J. (2007). Tu potencial emprendedor. México: Pearson.

Rebeil, C. M. \& Arevalo, M. R. (2017). Comunicación Integral para las Organizaciones Socialmente Responsables. En B. T. Ramírez y M. R. Arévalo (coords.), Comunicación Sustentable y Responsabilidad Social Empresarial (pp. 59-93). México: Tirant humanidades.

Rebeil, C. M. \& Ortiz, R. H. (2018). Relaciones Públicas y Responsabilidad Social Organizacional a través de medios digitales en apoyo a los afectados. El caso del sismo en la Ciudad de México de Septiembre de 2017. XIV Congreso de la Asociación Latinoamericana de Investigadores de la comunicación (ALAIC), Costa Rica. Recuperado de: https://www.alaic.org/site/wp-content/ uploads/2019/04/GT-2-ALAIC-2018_0.pdf

Rebeil, C. M., Hidalgo, T. J. \& Moreno, M. M. (2011). Gestión de la comunicación integrada enlas organizaciones: competenciasbásicas parala formación del gestor. En D. León (Coord.), Estudios de la comunicación: estrategias metodológicas y competencias profesionales en comunicación (pp. 51-82). México: Pearson Educación.

Rivas,M.A.(2005).Reseñade"LaPublicidadenelTercerSector.Tendenciasyperspectivas dela comunidad solidaria”. Convergencia. Revista de Ciencias Sociales, 12(37), 391399. Recuperado de: http://www.redalyc.org/articulo.oa?id=10503717

Rubio, G. J. (2007). El Tercer Sector frente a las transformaciones del estado del bienestar. Cuadernos de trabajo social, 20, 275-287.

Villafañe, J. (2004). La buena reputación: claves del valor intangible de las empresas. Madrid: Ediciones Pirámide.

\section{OTRAS REFERENCIAS}

Charity library (2018). Recuperado de: http://www.charitylibrary.co.uk/all-charities

Construyendo Organizaciones Civiles Transparentes, A.C(Confío)(2018). Recuperado de: http://confio.org.mx/inicio/osc-analizadas/ 
Fundación Lealtad (2018). Recuperado de: https://www.fundacionlealtad.org/ong/

Asociación de Directivos de Comunicación (DIRCOM) (2019). Recuperado de: http:// www.dircom.org/herramienta-2r

${ }^{*}$ Contribución: $100 \%$ de la autora.

* Nota: El editor Paul Capriotti y el Comité Editorial de la revista aprobaron la publicación del artículo.

\section{(cc) BY}

Artículo publicado en acceso abierto bajo la Licencia Creative Commons - Attribution 4.0 International (CC BY 4.0).

\section{IDENTIFICACIÓN DE LAAUTORA}

Herlinda Ortiz Rodríguez. Doctora en Alta Dirección por la Universidad de Altos Estudios Hispanoamericana, México. Magister en Comunicación Estratégica por la Benemérita Universidad Autónoma de Puebla (BUAP) y Licenciada en Publicidad y Relaciones Públicas por la Universidad Veracruzana (UV), México. Doctoranda en el doctorado de Investigación de la Comunicación, Universidad Anáhuac México. Investigadora, docente y consultora en áreas de comunicación, publicidad, diseño, relaciones públicas y mercadotecnia. Autora de productos de investigación a nivel nacional einternacional como: comunicación organizacional web de la ética en las organizaciones del tercer sector; análisis de modelos de relaciones públicas; comunicación integral en las organizaciones del tercer sector; relaciones públicas, reputación y responsabilidad social organizacional. Ha presentado diferentes ponencias y talleres a nivel nacional e internacional en congresos organizados por la Asociación Mexicana de Investigadores de la Comunicación (AMIC), la Asociación Latinoamericana de Investigadores de la Comunicación (ALAIC) y la Asociación Mexicana de Investigadores y Profesionales de la Comunicación Organizacional (AMIPCO).

\section{REGISTRO BIBLIOGRÁFICO}

Ortiz Rodríguez, H. (enero-junio, 2020). Comunicación Publicitaria en Medios Digitales. La gestión de la responsabilidad social en las Organizaciones del Tercer Sector. InMediaciones de la Comunicación, 15(1), 109-131. 